\title{
Wind Farm Energy and Costs Optimization Algorithm under Uncertain Parameters of Wind Speed Distribution
}

\author{
Vadim ROMANUKE \\ Polish Naval Academy, Faculty of Navigation and Naval Weapons, \\ 69 Śmidowicza Street, Gdynia, 81-127, Poland \\ v.romanuke@amw.gdynia.pl
}

\begin{abstract}
A problem of optimally projecting a wind farm is considered. The criteria are the energy produced by the farm and its costs. The starting option is either financial investments into the farm or a nominally planned energy. The wind speed distribution parameters are uncertain and given as interval estimates. In general, the farm consists of wind turbines having diverse power curves. An algorithm of maximizing the expected energy annually produced by them along with minimizing their costs is developed. If the investments are given, the energy is maximized in order not to outgo them under the uncertain parameters of wind speed distribution. Otherwise, if an annual energy is planned, the farm costs are minimized along with maximizing the produced energy, under the same uncertainty. The uncertainty is removed owing to finding the guaranteed and expected values of the costs and the wind farm power outputs. Depending on the initially given option (investments or an annual planned energy), the optimization efficiency is measured via the respective ratios.
\end{abstract}

Keywords: Wind farm, Wind turbine, Wind speed distribution, Uncertain parameters, Energy, costs, Optimization.

\section{Introduction}

\subsection{Problems of wind energy}

Wind power is the most promising direction of energy generation. Wind farm capacities increase nearly exponentially. Offshore wind farms will grow the most further while offshore wind power capacity is expected to reach a total of $75 \mathrm{GW}$ worldwide by 2020 , with significant contributions from China and the US. The wind power industry sets new records annually.

Electric power generated from wind power can be highly variable at several different timescales: hourly, daily, or seasonally. Annual variation also exists, but is not so significant. Because instantaneous electrical generation and consumption must remain in balance to maintain grid stability, this variability hinders from incorporating large amounts of wind power into a grid system $[2,5]$.

Mostly, a wind farm (WF) is a group of identical wind turbines (WTs) in the same location. A large WF may consist of several hundred WTs and cover an extended area of hundreds of square miles. Thus, another problem is that a WT effectively generates energy in a narrow band of wind speed, whereas wind speed is very volatile even over small areas. Both maintaining grid stability and increasing WF effectiveness are a challenge for wind power improvement $[2,3,8]$.

\subsection{Background and motivation}

Except for a number of WTs, an average wind power capacity strongly depends on wind statistics of an area, whereon a WF of those WTs is projected. Wind speeds in most of the world are modeled using the Weibull distribution [8]:

$$
\begin{aligned}
& p(s, b, a)=(b / a) \cdot(s / a)^{b-1} e^{-(s / a)^{b}} \text { by } \\
& \int_{0}^{\infty} p(s, b, a) d s=1,
\end{aligned}
$$

where $s$ is the wind speed, $b$ is the shape parameter related to the range of wind speed variations, and $a$ is the scale parameter related to the mean wind speed. This distribution tells us how often winds of different speeds are registered at a location with a certain average (mean) wind speed. Knowing this helps in selecting a WT with a rational cut-in speed (the wind speed, at which the turbine starts to generate usable power) and cut-out speed (the speed, at which the turbine hits the limit of its alternator and does not generate energy with further increases in wind speed), constituting the respective power curve $[2,9,6]$. However, such selection is hardly effective as a typical Weibull distribution of wind statistics is far narrower than the power curve (see Figure 1), where $w(s)$ is a power generated with a WT at the speed $s[9$, 4]. Indeed, the expected power generated with a WT by non-gusty winds is an integral

$\int_{0}^{\infty} w(s) p(s, b, a) d s$

Note that the mean of wind speed and its most frequent magnitude are less than the rated-out 
speed. They are closer only for smaller WTs having smaller blades $[2,8,6]$. Eventually, the product $w(s) p(s, b, a)$ becomes a pulse-like curve and thus integral (2) does not give a great value that might be anticipated.

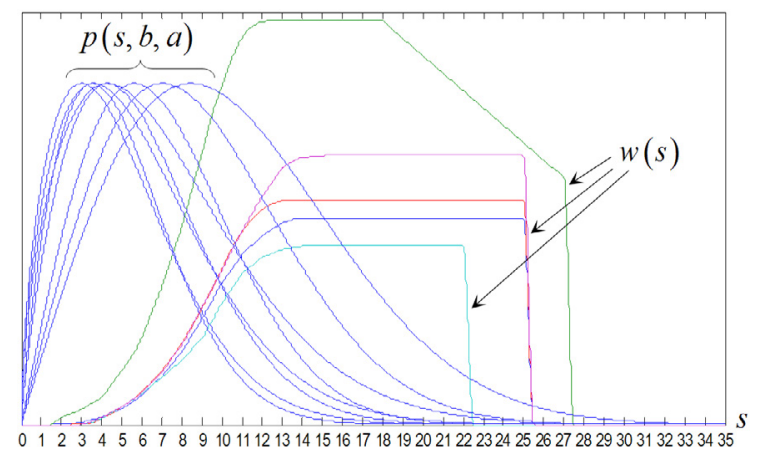

Figure 1. Comparison of a few typical Weibull normalized distributions of wind statistics and power curves of five real WTs

(available at www.thewindpower.net/data/samples)

An ideal case is that when power curve coincides with wind statistics. This is non-achievable in practice. However, some papers suggest that wind statistics may be met by using novel improvements in construction of WTs $[6,7,10]$. In particular, article [7] proposes a novel pitch control method that integrates a non-standard extended Kalman filter-based estimator, where a cascade control structure is developed for the pitch controller receiving the speed reference from a power-speed scheduler. Article [10] introduces a novel dual-rotor WT concept with an auxiliary upwind rotor and a bigger downwind rotor. More in a theoretical sense, article [1] presents a fuzzy duration forecast model for the WT construction project subject to the impact of wind uncertainty. Obviously, such approaches and similar ones require big investments for mass production. So, another optimistic way is to cover the wind statistics with multiple WTs, each of which holds its narrow band of wind speeds $[8,3]$. In this way, a WF is projected and built without construction inventions but just with allocating and using WTs optimally. The optimality here is understood as maximization of the expected generated by a WF. In other words, a sum of integrals of type (2) for multiple WTs is to be maximized. However, parameters $a$ and $b$ in those integrals can be confidently estimated only as intervals that makes the wind speed distribution (WSD) uncertainty.
Along with increasing the energy produced by WFs, economical reasonability is another strong factor. WF projects are required not to be very expensive, unless investors will just retreat. Therefore, the expected power generated with the WF turbines should be maximized by simultaneously minimizing costs of the WF. But from the side of investors, it would be very comfortable to give an amount of financial resources which could ensure a close-to-maximum of annual energy produced by the WF. On the other hand, if initially a planned annual energy is given then the costs for achieving this energy should be as minimal as possible. Here, the achievement implies that such energy cannot be produced exactly. Thus, along with the (nominally) planned energy, a minimum of the energy is given. The factually produced energy should not be less than this minimum. These particular problems motivate to develop a whole algorithm of WF energy and costs optimization under the WSD uncertainty.

\subsection{Goal and tasks for its achievement}

As there is no complete model of projecting a WF by the given planned energy and investments, the goal is to develop an algorithm of maximizing the expected produced energy (EPE) on a period of time by minimizing the costs. The WSD uncertainty should be controlled in the sense of guaranteeing the best result. The best result is either a maximized energy or minimized costs. For achieving this goal, the following tasks are to be accomplished:

1. To denote and explain generally those values, variables, notions, conventions, and abbreviations that will be used.

2. To state a problem of maximizing EPE by a given amount of financial resources under interval estimates of $a$ and $b$.

3. To state a problem of minimizing costs in order to achieve a planned annual energy under interval estimates of $a$ and $b$.

4. To unite those two problems into a single one, and describe a method of solving it algorithmically.

5. To exemplify the method in real WTs. 


\section{General denotations}

\subsection{Denotations of energy and investments}

Let the period of EPE maximization be equal to one year. Then denote the annual nominally planned energy (ANPE) by $E_{0}$, in MWh $[2,5]$. While maximizing, magnitude $E_{0}$ is unlikely to be achieved exactly, so a minimal ANPE is given. Denote the minimal ANPE by $E_{\min }$. Obviously, EPE denoted by $\tilde{E}$ cannot be less than the minimal ANPE:

$$
E_{\min } \leqslant \tilde{E} \leqslant E_{0} \text {. }
$$

Then a problem is to minimize costs of a WF project along with maximizing EPE under constraint (3). Inequality $\tilde{E}>E_{0}$ is excluded because a surplus energy must be subsequently utilized, sold, or stored, that is not always easy and it takes costs as well.

An amount of financial resources $C_{0}$, that could be called the initial farm investments (IFIs) into a WF project, cannot be given simultaneously with $E_{0}$ and $E_{\min }$ because then both energy maximization and cost minimization may probably fail. A subgoal here is to spend no more than $C_{0}$ along with maximizing the EPE. Now, it is not about to minimize the costs but to most efficiently use the invested resources.

\subsection{A configuration of WF by types of its WTs}

Theoretically, a WF may consist of a single WT. If a WF consist of multiple WTs, their types may be various depending on which ANPE or IFIs are given and what wind statistics of the area are. Let $T$ be a number of different types of WTs, where $T \in \mathbb{N}$. A formal case $T=1$ cannot be excluded as a WF consisting of identical WTs (ITWF) may perform theoretically better than a WF consisting of at least two different WTs (DTWF). A total number of WTs, which configure either an ITWF or a DTWF, depends on only which ANPE or IFIs are given.

A way of how a WF is physically configured, that is what distances between WTs and their positioning are, is implied by default to be close to a perfect one. The physical configuration of WFs does really influence on their performance, but WTs are positioned in their optimal way independently of optimizing EPE and costs (or efficiency of the invested resources). An optimal positioning of WTs is done only after the optimization is accomplished, because only then the number of WTs and their types are known.

\subsection{Expected powers of ITWF and DTWF}

Let $w(t, s)$ be a power generated with WT of type $t$ at speed $s$. It is the WT power curve. Then, given wind statistics by its parameters $a$ and $b$ for distribution (1), the expected power output (EPO) produced by this WT is

$$
m(t ; b, a)=\int_{0}^{\infty} w(t, s) p(s, b, a) d s
$$

If an ITWF consists of $q\left(t_{1}\right)=Q$ WTs of type $t_{1}$ by $t_{1} \in\{\overline{1, T}\}$ and $Q \in \mathbb{N}$, then EPO of the ITWF is $r\left(t_{1}, Q ; b, a\right)=Q \cdot m\left(t_{1} ; b, a\right)=$

$=Q \cdot \int_{0}^{\infty} w\left(t_{1}, s\right) p(s, b, a) d s$

In general, a DTWF is of $K$ different types of the WT, where $K \in\{\overline{2, T}\}$ for $T \in \mathbb{N} \backslash\{1\}$. Each WT type can be positioned/installed its own number of times (this is a number of sites/places where WTs of this type are installed). Let a subset of those types be $\left\{t_{k}\right\}_{k=1}^{K} \subset\{\overline{1, T}\}$. If WT of the $t_{k}$-th type is installed in $q\left(t_{k}\right)$ sites, where $q\left(t_{k}\right) \in \mathbb{N}$, then EPO of the DTWF is

$r\left(\left\{t_{k}\right\}_{k=1}^{K},\left\{q\left(t_{k}\right)\right\}_{k=1}^{K} ; b, a\right)=\sum_{k=1}^{K} q\left(t_{k}\right) m\left(t_{k} ; b, a\right)=$

$=\sum_{k=1}^{K} q\left(t_{k}\right) \int_{0}^{\infty} w\left(t_{k}, s\right) p(s, b, a) d s$

It is clear that for an ITWF, when $K=1$, EPO (6) becomes EPO (5). Thus, sum (6) is a general statement for calculating EPO.

\subsection{Transfer from ANPE and EPE to EPO}

As EPO (6) is measured in megawatts (MW), an annual EPO is found from ANPE which is divided by all the annual active hours. We count that the WF and its WTs work without interruptions, so there are $24 \cdot 365.25=8766$ active hours in a year. Then, given an ANPE $E_{0}$ and a minimal ANPE $E_{\min }$, their corresponding EPOs are

$r_{0}=E_{0} / 8766, r_{\min }=E_{\min } / 8766$.

For transferring back from an EPO to the corresponding EPE, we will just multiply sum (6) by 8766 . 


\subsection{Costs for buying and installing the WT}

Costs for buying and installing the WT are generally different. So let $g(t)$ be a cost of buying the WT of type $t$, and let $h(t)$ be a cost of installing this WT. Before setting IFIs $C_{0}$, costs $\{g(t)\}_{t=1}^{T}$ and $\{h(t)\}_{t=1}^{T}$ should be taken into consideration. This is a condition

$C_{0} \geqslant \max _{t=1, T}[g(t)+h(t)]$

that allows us to include those $T$ different types of WTs for a theoretical possibility of using at least one of them. When inequality (8) is false, then the WTs that are too expensive are to be excluded, but an effective DTWF can hardly be built with such spare IFIs. The condition of admissible costs for an ITWF is

$Q \cdot\left[g\left(t_{1}\right)+h\left(t_{1}\right)\right] \leqslant C_{0}$

and

$\sum_{k=1}^{K} q\left(t_{k}\right)\left[g\left(t_{k}\right)+h\left(t_{k}\right)\right] \leqslant C_{0}$

is the condition of admissible costs for a DTWF. Clearly, the admissibility is determined by IFIs.

\section{Maximization of EPE by IFIs}

\subsection{EPO maximum at fixed $a$ and $b$}

Assume that point estimates for parameters $a$ and $b$ are known (fixed). Then a problem of maximizing EPE by a given $C_{0}$ is equivalent to finding

$$
\begin{aligned}
& \max _{\left\{q\left(t_{k}\right)_{k=1}^{K} \max _{k=1}\right\}_{k=1}^{K} \in\{1, T\}} r\left(\left\{t_{k}\right\}_{k=1}^{K},\left\{q\left(t_{k}\right)\right\}_{k=1}^{K} ; b, a\right)= \\
& =r\left(\left\{t_{k}^{*}\right\}_{k=1}^{K_{*}},\left\{q_{*}\left(t_{k}^{*}\right)\right\}_{k=1}^{K_{*}} ; b, a\right)=r_{*}(b, a)
\end{aligned}
$$

and determining those numbers $\left\{q_{*}\left(t_{k}^{*}\right)\right\}_{k=1}^{K_{*}}$ for a subset $\left\{t_{k}^{*}\right\}_{k=1}^{K_{*}} \subset\{\overline{1, T}\}$ at which maximal EPO (11) is achieved, where condition

$\sum_{k=1}^{K_{*}} q_{*}\left(t_{k}^{*}\right)\left[g\left(t_{k}^{*}\right)+h\left(t_{k}^{*}\right)\right] \leqslant C_{0}$

should not be broken. Those integers are stored for every couple $a$ and $b$, so denote them by

$\left\{t_{k}^{*}(b, a)\right\}_{k=1}^{K_{*}(b, a)},\left\{q_{*}\left(t_{k}^{*}(b, a)\right)\right\}_{k=1}^{K_{*}(b, a)}$.

Here the task of minimizing the WF costs is not formulated explicitly. It just means that we must spend no more than amount $C_{0}$.

\subsection{The guaranteed EPO maximum}

EPO maximum $r_{*}(b, a)$ by (11) is uncertain while parameters $a$ and $b$ are evaluated as intervals, although these intervals may be pretty much narrow. Let $a \in\left[a_{1} ; a_{2}\right]$ and $b \in\left[b_{1} ; b_{2}\right]$. Practically, interval $\left[b_{1} ; b_{2}\right]$ of possible shape parameter values is a way narrower than interval $\left[a_{1} ; a_{2}\right]$ of possible scale parameter values as the mean wind speed varies badly. For instance (of a definite area), for an average shape parameter $\tilde{b}=1.7$ an interval estimate can be $\left[b_{1} ; b_{2}\right]=[1.675 ; 1.725]$ and an interval estimate $\left[a_{1} ; a_{2}\right]=[7.5 ; 9.5]$ would be for an average scale parameter $\tilde{a}=8$.

Turning back to uncertain EPO maximum (11), value $r_{* *}=\min _{a \in\left[a_{1} ; a_{2}\right]} \min _{b \in\left[b_{1} ; b_{2}\right]} r_{*}(b, a)$

is the guaranteed EPO maximum. It implies that we will obtain EPO at least equal to (14). A real obtained EPO will be likely greater than (14), but a relative difference between them is small if intervals $\left[a_{1} ; a_{2}\right]$ and $\left[b_{1} ; b_{2}\right]$ are relatively narrow.

\subsection{An expected maximum of EPO}

Assuming that probabilities of both parameters from their intervals $\left[a_{1} ; a_{2}\right]$ and $\left[b_{1} ; b_{2}\right]$ are distributed uniformly, we can find an expected maximum of EPO

$\overline{r_{* *}}=\frac{1}{\left(b_{2}-b_{1}\right)\left(a_{2}-a_{1}\right)} \int_{a_{1}}^{a_{2} b_{2}} \int_{b_{1}} r_{*}(b, a) d b d a$

Our assumption will be valid if those intervals are relatively narrow. In practice, the intervals are taken as finite subsets covering the infinite sets uniformly:

$$
A=\left\{a^{\langle i\rangle}\right\}_{i=0}^{N_{a}}=\left\{a_{1}+\frac{a_{2}-a_{1}}{N_{a}} i\right\}_{i=0}^{N_{a}} \subset\left[a_{1} ; a_{2}\right]
$$

by $\quad a^{\langle 0\rangle}=a_{1}, \quad a^{\left\langle N_{a}\right\rangle}=a_{2}$,

$$
B=\left\{b^{\langle j\rangle}\right\}_{j=0}^{N_{b}}=\left\{b_{1}+\frac{b_{2}-b_{1}}{N_{b}} j\right\}_{j=0}^{N_{b}} \subset\left[b_{1} ; b_{2}\right]
$$

by $b^{\langle 0\rangle}=b_{1}, \quad b^{\left\langle N_{b}\right\rangle}=b_{2}$.

Owing to (16) and (17), by the trapezoid rule we have the following approximations:

$$
\begin{aligned}
& \int_{b_{1}}^{b_{2}} r_{*}(b, a) d b \approx \\
& \approx \frac{b_{2}-b_{1}}{2 N_{b}}\left(r_{*}\left(b_{1}, a\right)+2 \sum_{j=1}^{N_{b}-1} r_{*}\left(b^{\langle j\rangle}, a\right)+r_{*}\left(b_{2}, a\right)\right)
\end{aligned}
$$


and

$\int_{a_{1}}^{a_{2}} r_{*}(b, a) d a \approx$

$\approx \frac{a_{2}-a_{1}}{2 N_{a}}\left(r_{*}\left(b, a_{1}\right)+2 \sum_{i=1}^{N_{a}-1} r_{*}\left(b, a^{\langle i\rangle}\right)+r_{*}\left(b, a_{2}\right)\right)$.

Then expected maximum of EPO (15) is approximately found as

$\overline{r_{* *}} \approx \frac{1}{4 N_{a} N_{b}}\left(r_{*}\left(b_{1}, a_{1}\right)+2 \sum_{i=1}^{N_{a}-1} r_{*}\left(b_{1}, a^{(i\rangle}\right)+r_{*}\left(b_{1}, a_{2}\right)+\right.$

$+2 \sum_{j=1}^{N_{b}-1}\left[r_{*}\left(b^{\langle j\rangle}, a_{1}\right)+2 \sum_{i=1}^{N_{a}-1} r_{*}\left(b^{\langle j\rangle}, a^{\langle i\rangle}\right)+r_{*}\left(b^{\langle j\rangle}, a_{2}\right)\right]+$

$\left.+r_{*}\left(b_{2}, a_{1}\right)+2 \sum_{i=1}^{N_{a}-1} r_{*}\left(b_{2}, a^{\langle i\rangle}\right)+r_{*}\left(b_{2}, a_{2}\right)\right)$.

With finite sets (16) and (17), guaranteed EPO maximum (14) is factually found as

$r_{* *} \approx \min _{i=0, N_{a}} \min _{j=0, N_{b}} r_{*}\left(b_{1}+\frac{b_{2}-b_{1}}{N_{b}} j, a_{1}+\frac{a_{2}-a_{1}}{N_{a}} i\right)$

but a difference between (14) and (19) will be null if function $r_{*}(b, a)$ is monotonic. The potential monotonicity of function $r_{*}(b, a)$ gives that double minimum is achieved at one of the corners of rectangle

$\left\{a_{1}+\frac{a_{2}-a_{1}}{N_{a}} i\right\}_{i=0}^{N_{a}} \times\left\{b_{1}+\frac{b_{2}-b_{1}}{N_{b}} i\right\}_{j=0}^{N_{b}} \subset$

$\subset\left[a_{1} ; a_{2}\right] \times\left[b_{1} ; b_{2}\right]$.

It depends on the wind statistics of the selected area which of two EPO maxima to choose. Guaranteed EPO maximum (19) must be chosen for a case of poor statistics. Otherwise, expected maximum of EPO (18) is chosen when the wind statistics are sufficient to make pretty-narrowinterval estimations of shape and scale parameters of the WSD.

\subsection{The best configuration of WF by IFIs}

We have two versions of the point estimation of the EPO maximum: the factual guaranteed maximum by (19) and the expected one by (18). Whichever point estimation of the EPO maximum is chosen, the best WF configuration is selected by a criterion of proximity of the point estimation and values of set

$\left\{\left\{r_{*}(b, a)\right\}_{a \in A}\right\}_{b \in B}$.

A value of set (21) being closest to the EPO maximum point estimation gives us integers $\left\{t_{k}^{* *}\right\}_{k=1}^{K_{* *}}$ and $\left\{q_{* *}\left(t_{k}^{* *}\right)\right\}_{k=1}^{K_{* *}}$

that constitute the best WF configuration.

\section{Minimization of costs by ANPE}

\subsection{WF cost minimum at fixed $a$ and $b$}

Henceforward, for further simplification, let EPO (6) be denoted by $r_{z}(b, a)$ subscripted with a corresponding index $z$ showing a definite combination version of WT types and their numbers. When ANPE is given first, we determine a finite subset

$R=\left\{\tilde{r}_{z}(b, a)\right\}_{z=\overline{1, Z}}$

of those EPOs (6) that satisfy a condition

$r_{z}(b, a) \in\left[r_{\min } ; r_{0}\right]$.

If $R=\varnothing$ then it means that the slot between the given ANPE and the minimal ANPE is likely to be too small. The same is concluded if $|R|=1$ or $|R|=2$. Then either ANPE must be re-given greater or its minimal value $E_{\min }$ must be set lesser. Another option is to correct both of them.

The $z$-th EPO $\tilde{r}_{z}(b, a)$ in set (23) is obtained by a WF consisted of $K_{z}$ different types of the WT, with a subset $\left\{t_{k}^{\langle z\rangle}\right\}_{k=1}^{K_{z}} \subset\{\overline{1, T}\}$ of those types. The WT of the $t_{k}^{\langle z\rangle}$-th type is installed in $q_{z}\left(t_{k}^{\langle z\rangle}\right)$ sites. Such WF is a DTWF if $K_{z}>1$ and it is an ITWF for $K_{z}=1$. Amount

$c_{z}(b, a)=\sum_{k=1}^{K_{z}} q_{z}\left(t_{k}^{\langle z\rangle}\right)\left[g\left(t_{k}^{\langle z\rangle}\right)+h\left(t_{k}^{\langle z\rangle}\right)\right]$

represents the costs of obtaining the $z$-th EPO $\tilde{r}_{z}(b, a)$ in set (23). A set of all the costs corresponding to set (23) is $\left\{c_{z}(b, a)\right\}_{z=\overline{1, Z}}$. A question is how to determine the costs that would correspond to EPOs (7). For this, it is natural to assume that the cost distribution is quasilinear. Thus a proportion between the costs and EPO must hold. Hence, the formal costs corresponding to EPO $r_{0}$ are

$c_{0}(b, a)=\frac{\max _{z=1, Z} c_{z}(b, a)}{\max _{z=1, Z} \tilde{r}_{z}(b, a)} \cdot r_{0}$

Similarly, the formal costs corresponding to EPO $r_{\text {min }}$ are 
$c_{\min }(b, a)=\frac{\min _{z=1, Z} c_{z}(b, a)}{\min _{z=1, Z} \tilde{r}_{z}(b, a)} \cdot r_{\min }$

Subsequently, costs (25) and EPOs in set (23) are standardized:

$\lambda_{z}(b, a)=\frac{c_{z}(b, a)-c_{\min }(b, a)}{c_{0}(b, a)-c_{\min }(b, a)}$,

$\theta_{z}(b, a)=\frac{r_{0}-\tilde{r}_{z}(b, a)}{r_{0}-r_{\min }}$

The WF cost minimum at fixed $a$ and $b$ is achieved at point

$z_{*} \in \arg \min _{z=1, Z} \lambda_{z}(b, a)$

On the other hand, EPO can be maximized to reach out for ANPE by minimizing the distance between $r_{0}$ and $\tilde{r}_{z}(b, a)$ :

$z^{*} \in \arg \min _{z=1, Z} \theta_{z}(b, a)$

Obviously, problems (30) and (31) constitute a two-criterion problem wherein its solutions $z_{*}$ and $z^{*}$ are usually different. In other words, minimum in (30) and minimum in (31) are achieved at different combination versions of WT types and their numbers. Therefore, the two-criterion problem is solved with an efficient solution (to a single-criterion problem)

$z_{\mathrm{PE}} \in \arg \min _{z=1, Z} \sqrt{\lambda_{z}^{2}(b, a)+\theta_{z}^{2}(b, a)}$

that is the closest-to-zero point. In fact, point $z_{\mathrm{PE}}$ by (32) gives both costs $c_{z_{\mathrm{PE}}}(b, a)$ closest to $c_{\text {min }}(b, a)$ and EPO $\tilde{r}_{z_{\mathrm{PE}}}(b, a)$ closest to $r_{0}$. The corresponding integers (13) are stored.

\subsection{The guaranteed minimum of WF costs}

The guaranteed minimum of WF costs is factually found as

$$
c^{*} \approx \max _{i=0, N_{a}} \max _{j=0, N_{b}} c_{z_{\mathrm{PE}}}\left(b_{1}+\frac{b_{2}-b_{1}}{N_{b}} j, a_{1}+\frac{a_{2}-a_{1}}{N_{a}} i\right)
$$

with finite sets (16) and (17). It means that building a WF will cost us no more than amount resulted from (33).

\subsection{An expected minimum of WF costs}

The real costs in comparison to (33) are expected to be slightly lesser. An expected minimum of WF costs $\bar{c}^{*}=\frac{1}{\left(b_{2}-b_{1}\right)\left(a_{2}-a_{1}\right)} \int_{a_{1}}^{a_{2}} \int_{b_{1}}^{b_{2}} c_{z_{\mathrm{PE}}}(b, a) d b d a$

is estimated similarly to approximation (18). Costs (34) expectedly allow obtaining the best EPO

$\overline{\tilde{r}}^{*}=\frac{1}{\left(b_{2}-b_{1}\right)\left(a_{2}-a_{1}\right)} \int_{a_{1}}^{a_{2}} \int_{b_{1}}^{b_{2}} \tilde{r}_{z_{\mathrm{PE}}}(b, a) d b d a$

by the same approximation scheme. The best EPO is guaranteed at level

$\tilde{r}^{*} \approx \min _{i=0, N_{a}} \min _{j=0, N_{b}} \tilde{r}_{\mathrm{PF}_{\mathrm{PE}}}\left(b_{1}+\frac{b_{2}-b_{1}}{N_{b}} j, a_{1}+\frac{a_{2}-a_{1}}{N_{a}} i\right)$

with finite sets (16) and (17). Costs for either EPO (36) or EPO (35) do not exceed guaranteed costs (33).

\subsection{The best configuration of WF by ANPE}

The best WF configuration as integers (22) here is selected by a criterion of proximity of the twocomponent point estimation of the best efficient costs-and-EPO and values of set

$\left\{\left\{\left[c_{z_{\mathrm{PE}}}(b, a) \quad \tilde{r}_{z_{\mathrm{PE}}}(b, a)\right]\right\}_{a \in A}\right\}_{b \in B}$.

The two-component point estimation is either $\left[\begin{array}{cc}c^{*} & \tilde{r}^{*}\end{array}\right]$ or $\left[\begin{array}{ll}\bar{c}^{*} & \overline{\tilde{r}}^{*}\end{array}\right]$ by formulae (33) - (36). The proximity is implied by the minimal Euclidean distance

$\sqrt{\left(u_{1}(b, a)-u_{1}^{*}\right)^{2}+\left(v_{1}(b, a)-v_{1}^{*}\right)^{2}}$

between points $\left[u_{1}(b, a) v_{1}(b, a)\right]$ and $\left[u_{1}^{*} v_{1}^{*}\right]$, where costs and EPOs are normalized by rules

$x_{1}(b, a)=x(b, a) / \max _{a \in A} \max _{b \in B} x(b, a)$,

$x_{1}^{*}=x^{*} / \max _{a \in A} \max _{b \in B} x(b, a)$

Integers (22) correspond to a minimal distance by (38).

\section{Algorithm of optimization}

Once a decision on what is to be optimized is made, a person responsible for the WF project must learn costs of various WTs. While learning, inequality (8) is considered for really admissible IFIs. On this basis a set of $T$ different WTs is formed. For speeding up the optimization process, we should put some constraints. When $C_{0}$ is given, we find integer

$Q_{\max }=\rho\left(C_{0} / \min _{t=1, T}[g(t)+h(t)]\right)+1$, 
where function $\rho(x)$ rounds $x$ to the nearest integer less than or equal to $x$. When ANPE $E_{0}$ and a minimal ANPE $E_{\min }$ are given, it is

$$
Q_{\max }=\rho\left(r_{0} / \min _{t=1, T} m(t ; b, a)\right)+1 \text {. }
$$

Now, the wind farm energy and costs are optimized by the following algorithm:

1. Define number $T$.

2. Define intervals $\left[a_{1} ; a_{2}\right]$ and $\left[b_{1} ; b_{2}\right]$.

3. Break these intervals equidistantly into $N_{a}$ and $N_{b}$ tiny subintervals, respectively.

4. For every single $a$ from finite set (16) and every single $b$ from finite set (17) do:

5. Calculate $\operatorname{EPO}(4) \forall t=\overline{1, T}$.

6. If $C_{0}$ is given then do subroutine \#1, else, for the given ANPE $E_{0}$ and minimal ANPE $E_{\text {min }}$, do subroutine \#2.

Subroutine \#1:

s1. Calculate integer (41).

s2. Do ITWF-subroutine \#1.

s3. Do DTWF-subroutine \#1.

s4. Extract integers (13) at which maximal EPO (11) is achieved.

s5. End of subroutine \#1.

ITWF-subroutine \#1:

${ }^{\mathrm{I}} 1$. For every single $t_{1}=\overline{1, T}$ do:

2. For every single $Q=\overline{1, Q_{\max }}$ do:

3. If condition (9) holds, store $t_{1}, Q$, and EPO (5).

4. End of the loop started at item \#2.

I5. End of the loop started at item \#1.

${ }^{\mathrm{I}} 6$. End of ITWF-subroutine \#1.

DTWF-subroutine \#1:

D1. For every single $K=\overline{2, T}$ do:

D2. For every subset $\left\{t_{k}\right\}_{k=1}^{K} \subset\{\overline{1, T}\}$ do:
D3. For every set $\left\{q\left(t_{k}\right)\right\}_{k=1}^{K}$ do:

D4. If condition (10) holds, store $\left\{t_{k}\right\}_{k=1}^{K}$, $\left\{q\left(t_{k}\right)\right\}_{k=1}^{K}$ and EPO (6).

D5. End of the loop started at item \#3.

${ }^{\mathrm{D}} 6$. End of the loop started at item $\# 2$.

D7. End of the loop started at item \#1.

D8. End of DTWF-subroutine \#1.

Subroutine \#2:

s1. Calculate EPOs (7).

s2. Calculate integer (42).

s3. Do ITWF-subroutine \#2.

s4. Do DTWF-subroutine \#2.

s5. If $Z=0$ then return that there is no solution, else proceed.

${ }^{\mathrm{s}} 6$. Calculate costs (26) and (27).

s7. Based on costs (26) and (27), calculate standardizations (28) and (29) $\forall z=\overline{1, Z}$.

s8. Extract efficient point (32) of the uncertain best costs and EPO by the corresponding integers (13).

s9. End of subroutine \#2.

ITWF-subroutine \#2:

${ }^{\mathrm{I}} 1$. For every single $t_{1}=\overline{1, T}$ do:

2. For every single $Q=\overline{1, Q_{\max }}$ do:

3. If condition (24) holds, register the corresponding index $z$, store $t_{1}, Q$, and EPO $r_{z}(b, a)$ in (24) along with costs (25).

4. End of the loop started at item \#2.

5. End of the loop started at item \#1.

I6. End of ITWF-subroutine \#2.

DTWF-subroutine \#2:

${ }^{\mathrm{D}} 1$. For every single $K=\overline{2, T}$ do:

D2. For every subset $\left\{t_{k}\right\}_{k=1}^{K} \subset\{\overline{1, T}\}$ do: 
D3. For every set $\left\{q\left(t_{k}\right)\right\}_{k=1}^{K}$ do:

D4. If condition (10) holds, store $\left\{t_{k}\right\}_{k=1}^{K}$, $\left\{q\left(t_{k}\right)\right\}_{k=1}^{K}$ and EPO (6).

D5. End of the loop started at item \#3.

${ }^{\mathrm{D}} 6$. End of the loop started at item $\# 2$.

D7. End of the loop started at item \#1.

D8. End of DTWF-subroutine \#2.

7. End of the loop started in item \#4 of the main routine.

8. If $C_{0}$ was given then select from set (21) an EPO maximum which is closest to value (19) and to value (18), respectively, whereupon return the corresponding integers (22); otherwise, for the given ANPE $E_{0}$ and minimal ANPE $E_{\text {min }}$, then select from set (37) a couple which is closest to couple $\left[c^{*} \tilde{r}^{*}\right]$ and to couple $\left[\bar{c}^{*} \overline{\tilde{r}}^{*}\right]$, respectively, whereupon return the corresponding integers (22).

The optimization efficiency can be measured via the respective to subroutines \#1 and \#2 ratios

$$
\begin{aligned}
& \mu\left(E_{0}\right)=c_{\mathrm{IFIs}} / C_{0}, \bar{\mu}\left(E_{0}\right)=\bar{c}_{\mathrm{IFIs}} / C_{0}, \\
& e\left(E_{0}\right)=r_{\mathrm{ANPE}} / r_{0}, \bar{e}\left(E_{0}\right)=\bar{r}_{\mathrm{ANPE}} / r_{0},
\end{aligned}
$$

where $c_{\text {IFIs }}, r_{\mathrm{ANPE}}$ and $\bar{c}_{\mathrm{IFIs}}, \bar{r}_{\mathrm{ANPE}}$ are the costs and EPOs calculated at integers (22) extracted by the proximity of the guaranteed and expected values, respectively. It is not necessary that $\mu\left(E_{0}\right)<\bar{\mu}\left(E_{0}\right)$ or $e\left(E_{0}\right)<\bar{e}\left(E_{0}\right)$.

\section{Experimental examples}

Our experimental studies are carried out with 5 WTs: Enercon E82 E2 (2.3 MW), Gamesa G128-4.5 MW, Nordex N90/2500 (2.5 MW), REpower MM82 (2 MW), Vestas V112-3.0 MW. Their power curves are shown in Figure 1. Due to lack of reliable cost information, we assume their respective costs (in million euros) as

$$
\begin{aligned}
& g(1)=3.1, g(2)=7.72 \quad g(3)=3.25, g(4)=2.68 \\
& g(5)=5.1, h(t)=0.289 g^{2}(t) \quad \forall t=\overline{1,5} .
\end{aligned}
$$

The intervals of the WSD parameters are

$$
\left[a_{1} ; a_{2}\right]=[5.6 ; 6.75] \text { and }\left[b_{1} ; b_{2}\right]=[1.6 ; 1.8]
$$

that correspond to an average wind speed between 5 and $6 \mathrm{~m} / \mathrm{s}$. Intervals (46) are broken by $N_{a}=23$ and $N_{b}=8$. Figures 2 and 3 along with Table 1 show the results when ANPE is varied from 12,000 to $25,000 \mathrm{MWh}$ with a step $1,000 \mathrm{MWh}$, and the minimal ANPE is $90 \%$ of ANPE. Figures 4 and 5 along with Table 2 show the results when IFIs are varied from 14 to 22 million euros with a step 1 .

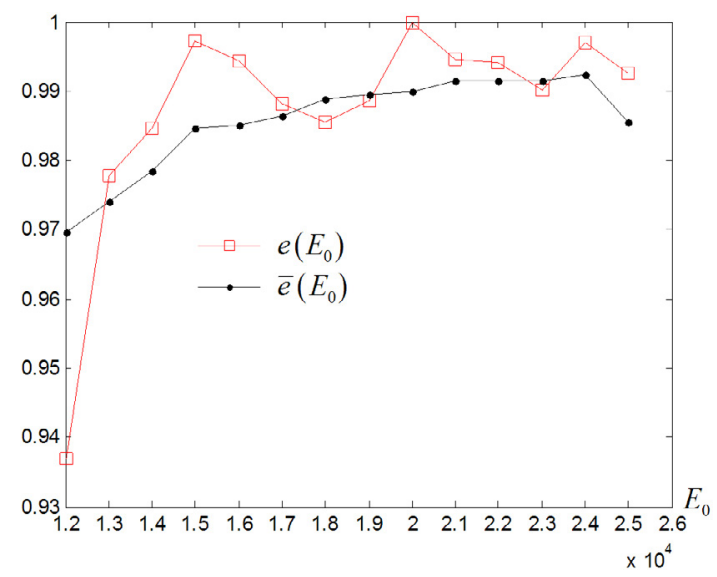

\begin{tabular}{|c|c|c|c|c|}
\hline \multirow[b]{2}{*}{$\begin{array}{c}E_{0}, \\
\text { MWh }\end{array}$} & \multicolumn{2}{|c|}{ Guaranteed } & \multicolumn{2}{|c|}{ Expected } \\
\hline & $\left\{t_{k}^{\text {fok }}\right\}_{k=1}^{K_{\text {*** }}}$ & $\left\{q_{* * *}\left(t_{k}^{\text {wpk }}\right)\right\}_{k=1}^{K_{* * *}}$ & $\left\{t_{k}^{* * *}\right\}_{k=1}^{K_{* *}}$ & $\left\{q_{* * *}\left(t_{k}^{\text {sph }}\right)\right\}_{k=1}^{K_{* *}}$ \\
\hline 12000 & 35 & 21 & 134 & 111 \\
\hline 13000 & 34 & 22 & 13 & 21 \\
\hline 14000 & 13 & 13 & 3 & 3 \\
\hline 15000 & 124 & $\begin{array}{lll}1 & 1 & 1\end{array}$ & 14 & 13 \\
\hline 16000 & 134 & 122 & 134 & 112 \\
\hline 17000 & 34 & 41 & 134 & 121 \\
\hline 18000 & 14 & 15 & 13 & 13 \\
\hline 19000 & 134 & 123 & 14 & 14 \\
\hline 20000 & 134 & 141 & 134 & 113 \\
\hline 21000 & 3 & 6 & 34 & 32 \\
\hline 22000 & 134 & 124 & 4 & 6 \\
\hline 23000 & 34 & 43 & 34 & 15 \\
\hline 24000 & 134 & 116 & 34 & 24 \\
\hline 25000 & 34 & 35 & 134 & 222 \\
\hline
\end{tabular}

Figure 2. Ratios (44) when ANPE is varied

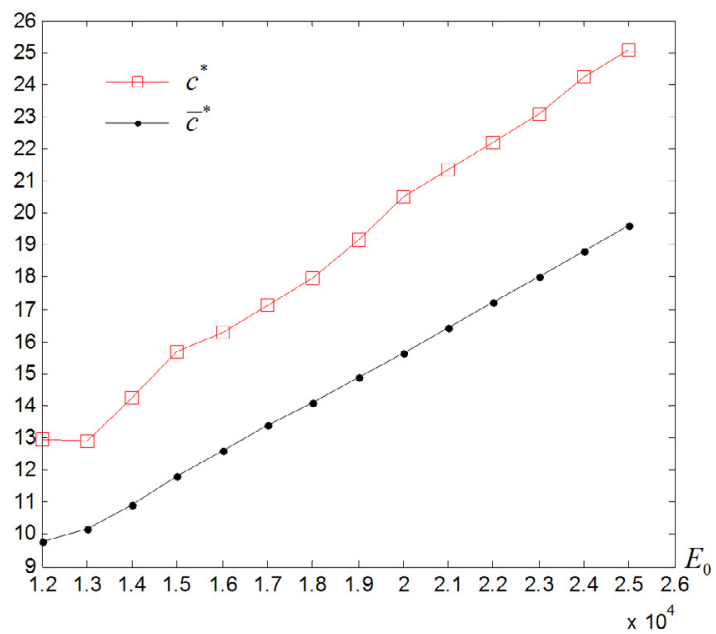

Figure 3. Guaranteed and expected costs to Figure 2

Table 1. Integers (22) to the results in Figures 2 and 3 


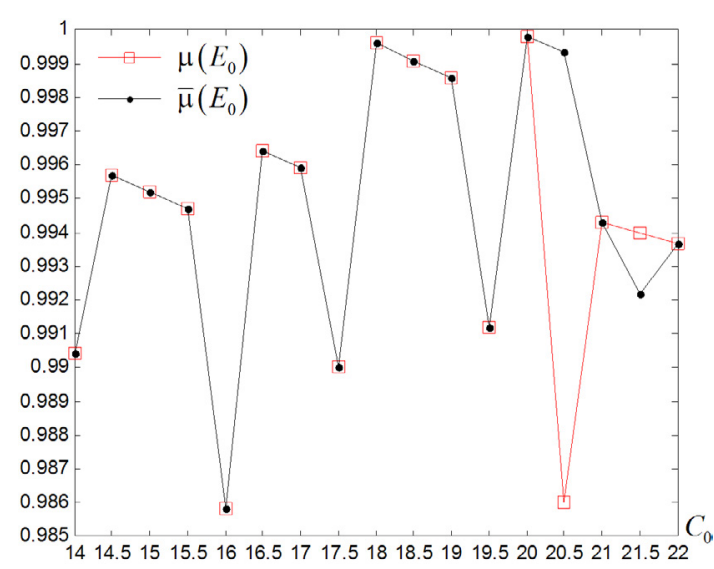

Figure 4. Ratios (43) when IFIs are varied

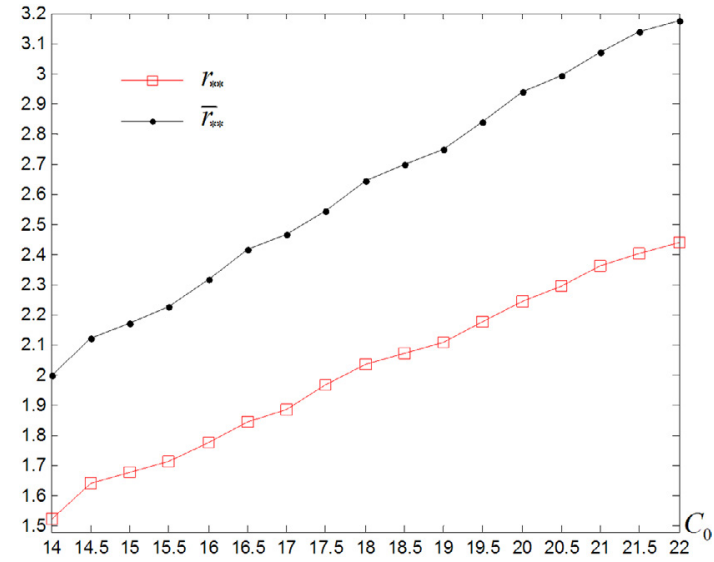

Figure 5. Guaranteed and expected EPOs to Figure 4

Table 2. Integers (22) to the results in Figures 4 and 5

\begin{tabular}{|c|c|c|c|c|}
\hline \multirow{2}{*}{\begin{tabular}{|c|}
$C_{0}$ \\
million \\
euros
\end{tabular}} & \multicolumn{2}{|c|}{ Guaranteed } & \multicolumn{2}{|c|}{ Expected } \\
\hline & $\left\{t_{k}^{* * k}\right\}_{k=1}^{K_{\text {wex }}}$ & $\left\{q_{* *}\left(t_{k}^{* *}\right)\right\}_{k=1}^{K_{* x}}$ & $\left\{t_{k}^{*}\right\}_{k=1}^{K_{* x}}$ & $\left\{q_{* *}\left(t_{k}^{* *}\right)\right\}_{k=1}^{K_{w *}}$ \\
\hline \begin{tabular}{|l|}
14 \\
\end{tabular} & 13 & 22 & 13 & 22 \\
\hline 14.5 & 4 & 5 & 4 & 5 \\
\hline 15 & 14 & 14 & 14 & 14 \\
\hline 15.5 & 14 & 23 & 14 & 23 \\
\hline 16 & 34 & 23 & 34 & 23 \\
\hline 16.5 & 34 & 32 & 34 & 32 \\
\hline 17 & 134 & 131 & 134 & 1331 \\
\hline 17.5 & 4 & 6 & & 6 \\
\hline 18 & 34 & 15 & 34 & 15 \\
\hline 18.5 & 134 & 114 & 134 & 114 \\
\hline 19 & 134 & 213 & 134 & 213 \\
\hline 19.5 & 34 & 33 & 34 & 33 \\
\hline 20 & 34 & 42 & 34 & 42 \\
\hline 20.5 & 4 & 7 & 134 & 141 \\
\hline 21 & 34 & 16 & 34 & 16 \\
\hline 21.5 & 134 & 115 & & 6 \\
\hline 22 & 134 & 214 & 134 & 214 \\
\hline
\end{tabular}

\section{Discussion}

Obviously, the results returned by the algorithm are not as exact as the given IFIs or ANPE. Some uncertainty remains in-between the guaranteed and expected values (like in Figures 2 and 4).
Moreover, the best WF configurations after integers (22) may differ (like in Tables 1 and 2). However, this is a natural fee of optimizing over rectangle (20).

The guaranteed EPO by a given ANPE can exceed the expected one as these EPOs are derived from simultaneously minimizing costs and distances between EPE and ANPE. Therefore, ratios (44) should not be analyzed separately. The closest couple of the ratios may be preferable but the highest ratios (either guaranteed or expected) are acceptable as well.

The optimization efficiency increases on average as ANPE is increased (see Figure 2). When IFIs are increased, the efficiency increment exists but it is more unstable (see Figure 4). Hence, if there is an uncertainty of selecting IFIs, a plot similar to Figure 4 must be drawn for finding the highest ratios (43). In the example, the best decision is to invest 20 million euros. However, investing 16 million euros by the worst case worsens the decision just for $1.4 \%$.

\section{Conclusions}

The developed algorithm allows an efficient distribution of the energy generated by WTs having diverse power curves. The power curve diversity is intentional to fit as ineffectiveness of the single WT against wind statistics, as well as to fit WSD uncertainty. If IFIs are given, the energy generated by a WF is maximized in order not to outgo the IFIs under uncertain parameters of WSD. Otherwise, if ANPE is given, a WF is built optimally in the sense of minimizing the costs of its WTs along with maximizing the produced energy, under the same uncertainty. The uncertainty is removed owing to finding the guaranteed and expected values of the costs and EPOs. Depending on the initially given option (IFIs or ANPE), the optimization efficiency is measured via respective ratios (43) and (44).

No solution can be returned by the algorithm in the cases when ANPE is set poorly. It is seen outright by that set (23) happens to be empty. Such an infeasibility is rectified by widening the slot between the given ANPE and the minimal ANPE. In the other cases, the algorithm allows converging to the best WF configuration, which produces an optimal energy by its optimal costs. 


\section{Acknowledgements}

The research reported in this paper has been supported by the Faculty of Navigation and Naval Weapons at Polish Naval Academy, Gdynia, Poland.

\section{REFERENCES}

1. Guo, S.-J., Chen, J.-H. \& Chiu, C.-H. (2017). Fuzzy duration forecast model for wind turbine construction project subject to the impact of wind uncertainty, Automation in Construction, 81, 401-410.

2. Letcher,T.M.(2017). Windenergyengineering. A handbook for onshore and offshore wind turbines. Elsevier, Academic Press.

3. Lungu, I., Căruţaşu, G., Pîrjan, A., Oprea, S.V. \& Bâra, A. (2016). A Two-step forecasting solution and upscaling technique for small size wind farms located in hilly areas of Romania, Studies in Informatics and Control, 25(1), 7786.

4. Lydia, M., Kumar, S. S., Selvakumar, A. I. \& Kumar, G. E. P. (2014). A comprehensive review on wind turbine power curve modeling techniques, Renewable and Sustainable Energy Reviews, 30, 452-460.
5. Ng, C. \& Ran, L. (2016). Offshore wind farms : technologies, design and operation. London, UK, Woodhead Publishing.

6. Pintea, A., Wang, H., Christov, N., Borne, P. \& Popescu, D. (2011). Modelling and power regulation of horizontal variable speed wind turbines, Studies in Informatics and Control, 20(3), 305312.

7. Song, D., Yang, J., Su, M., Liu, A., Cai, Z., Liu, Y. \& Joo, Y. H. (2017). A novel wind speed estimator-integrated pitch control method for wind turbines with global-power regulation, Energy, 138, 816-830.

8. Song, M., Wen, Y., Duan, B., Wang, J. \& Gong, Q. (2017). Micro-siting optimization of a wind farm built in multiple phases, Energy, 137, 95-103.

9. Villanueva, D. \& Feijóo, A. (2018). Comparison of logistic functions for modeling wind turbine power curves, Electric Power Systems Research, 155, 281-288.

10. Wang,Z., Ozbay,A., Tian, W. \& Hu, H. (2018). An experimental study on the aerodynamic performances and wake characteristics of an innovative dual-rotor wind turbine, Energy, 147, 94-109. 